\title{
Transgender Language: What Makes Turkish Male Homosexual Language Distinctive?
}

\author{
Meltem Sargın, Özgün Koşaner \\ Department of Linguistics, Dokuz Eylül University, İzmir, Turkey \\ Email:meltem.sargin@deu.edu.tr,ozgun.kosaner@deu.edu.tr
}

Received 14 March 2016; accepted 16 April 2016; published 19 April 2016

Copyright (C) 2016 by authors and Scientific Research Publishing Inc.

This work is licensed under the Creative Commons Attribution International License (CC BY). http://creativecommons.org/licenses/by/4.0/

\section{(c) (i) Open Access}

\begin{abstract}
Most gender-based linguistic studies in literature are related to men and women only, whereas the term gender is supposed to refer to transgender people as well. People whose sex and gender do not match (GLAAD, 2010) obviously use language in a different way from men and women in some aspects. This study aims to find out the acoustic and phonological differences of male homosexual language from those of men and women. Some male to female transgender people such as transsexuals and cross-dressers speak in a distinctive way, which usually becomes a criterion of judgment for stereotyping (Medhurst, 2002: pp. 314-315) and a matter of ridicule on the part of other people. The distinctiveness of this language use may result from acoustic, anatomical, hormonal, physiological and even philosophical differences (Pepiot, 2012). This study focuses on the acoustic and phonological aspects. To identify these aspects, voice recordings of male homosexuals were manipulated in the phonology laboratory and perception tests were conducted on a control group. The results of the study confirmed that one of the most important acoustic and phonological features that differentiate male homosexual speech from male and female heterosexual speech is pitch range, in other words intonation.
\end{abstract}

\section{Keywords}

Transgender Language, Homosexual Speech, Pitch Range, Intonation, Sociophonetics

\section{Introduction}

Although it is considered that the term gender encompasses transgenders - an umbrella term used to mean people whose biological and social sexes do not match (GLAAD, 2010)_as well as men and women, it is ob- 
served that most studies in the literature are related to only men and/or women. However, some transgender people use the language in a distinctively different way than men and women do for most of the time. Particularly, most man to woman transsexuals and transvestites and many man homosexuals speak in a significantly different way than heterosexual men and women, and this exaggerated way of speech with a concern to sound like a woman, which in fact makes them sound more, mostly becomes a criterion with reference to the norms of gender in society (Medhurst, 2002: pp. 314-315) and even a matter of mockery. In Islamic societies, in which transgenderism is not considered as a difference or variety but a sin or a deviance, this situation can reach to even more offending situations (Kugle 2010: p. 7).

Woman to man transgenders seem to be luckier than man to woman transgenders, since as a result of the intake of testosterone the vocal cords thickens and the voice deepens. On the other hand, man to woman transgenders do not have such a chance due to the fact that estrogen does not have any effect on the vocal cords, thus the voice pitch remains low (Kulick, 1999: p. 608). These people make a great afford to use language in accordance with their new gender, sometimes by undergoing surgeries which make their vocal cords shorter and tenser and by taking voice and speaking lessons. In order to be able to help these people it is necessary to know how they use language and in what way their language use is different from the language use of men and women in detail. When the language use of heterosexual, homosexual, and transsexual people is studied, it is seen that the differences of language use among these people result from acoustic, phonological, anatomical, hormonal, physiological and even philosophical reasons, and moreover it is observed that there are cross linguistic differences as well (Pepiot, 2012). This study focuses only on the acoustic and phonological ones out of the reasons mentioned above. The study proceeds as follows: first the aim and the hypothesis are presented. Later the methodology and the experiment design of the study are explained. The study is concluded with a discussion of findings.

\section{Aim of the Study}

The aim of this study is to conduct a preliminary study to find out the acoustic and phonetic differences that distinguish the language use of Turkish male homosexuals from heterosexual male and females. The hypothesis of the study is that one of the most important acoustic and phonological features that differentiate male homosexual speech from male and female heterosexual speech is pitch range, in other words intonation.

\section{Methodology}

The studies in the literature (Gaudio 1994; Linville 1998; Smyth, Jacobs, \& Rogers, 2003) generally appeal to perceptual evaluations and request the participants to predict to which gender and sexual orientation the voice they had listened belonged. However Levon (2006, 2007) criticises such a metrology asserting that this kind of methodology complicates controlling the variables. According to Levon this methodology has two main problems: 1) it places the identities under scrutiny at the centre of the study, and 2) it attempted to classify certain linguistic features as components of an identity/gender-special speech in a post hoc manner. This methodology first get sound recordings from speakers who identify themselves as members of some particular gender (including trangenders). Then these sound recordings are presented to the participants who are requested to rate the speakers in the recordings according to scales related to gender and sexuality. Then the researchers attempt to analyse the differences in the recordings belonging to different gender groups. This kind of a methodology assumes, a priori, that the behaviours of practices of a gender group will be internally homogenous, as well as mutually exclusive and maximally differentiated from another gender group (Levon, 2006: pp. 60-61). However, speakers tend to change the way they talk in different social contexts. In some of these contexts their sexual identities may be more salient than others.

Levon offers a different methodology to cope up with the problems summarized above. Instead of using raw sound recordings from different individuals belonging to different gender groups, he uses manipulated sound recordings from one individual. The sound recordings are manipulated with regard to sibilant duration and pitch range.

Following Levon (2006, 2007) we used manipulated sound recordings in our study, however in our study there is not only a single individual. The voice recordings were taken from five male heterosexuals $(\mathrm{N}=5)$, five male homosexuals $(\mathrm{N}=5)$ and five female heterosexuals $(\mathrm{N}=5)$. The number of the participants for each group was limitedto five, since it was difficult to find male homosexual participants due to the sensitivity of transgenderism and homosexuality in Turkish Society. The male homosexual participants were contacted via personal 
communication and selected randomly. The age and educational statuses of the participants were matched in all of the groups. All of the participants are older than 18 and either university students or university graduates. These individuals were requested to read a text passage comprising of 206 words. The text passage included 38 utterances, six of which were interrogative utterances to ensure a non-monotonous reading and to express different pitch contours. The voice recordings $(\mathrm{N}=15)$ were obtained at the Phonology Laboratory at Dokuz Eylül University Department of Linguistics, in an isolated and non-echoing room. The voice recordings were performed using the Isotope RX4 Advanced software.

Later these voice recordings were manipulated using the Time \& Pitch algorithm of Isotope RX4 Advanced, and male heterosexual sounds were converged towards female heterosexual sounds; female heterosexual sounds to male heterosexual sounds; and male homosexsual sounds to male heterosexual sounds with regard to F0 fundamental frequency and other formant values. Figure 1 shows the pitch contour of an original heterosexual male voice (blue contour) and the pitch contour of the pitch manipulated version of the same voice (green contour), which is converged towards female voice.

To manipulate the pitch range the Celemony Melodyne software was used. In this phase the voice recordings from male heterosexuals were manipulated to have a wider pitch range; recordings from female heterosexuals and male homosexuals to have a narrower pitch range. Figure 2 shows the manipulation of a heterosexual female voice. In Figure 2, the greenish fragments are the original female voice, the bluish fragments are the manipulated ones, and the darker fragments are the overlapping fragments, which are not altered. In this example the pitch range of the female voice is narrowed.

As a result of these manipulations the stimuli comprising of 15 original voice recordings, 15 voice recordings manipulated with regard to F0 fundamental frequency, and another 15 voice recordings manipulated with regard to both F0 fundamental frequency and pitch range. These 45 voice recordings were collected in three different data sets as presented in Table 1.

These data sets comprised the stimuli of the study and used were in the experimental design explained below.

\section{Experimental Design}

A series of experimental sets were constructed using 9 voice recordings, each from a different data set using

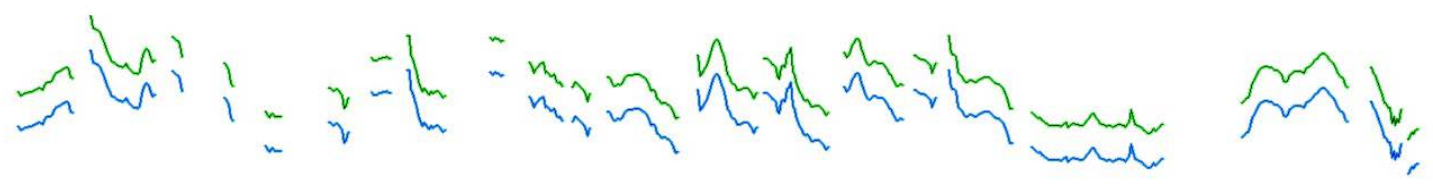

Figure 1. Pitch contours for original and pitch manipulated voices.

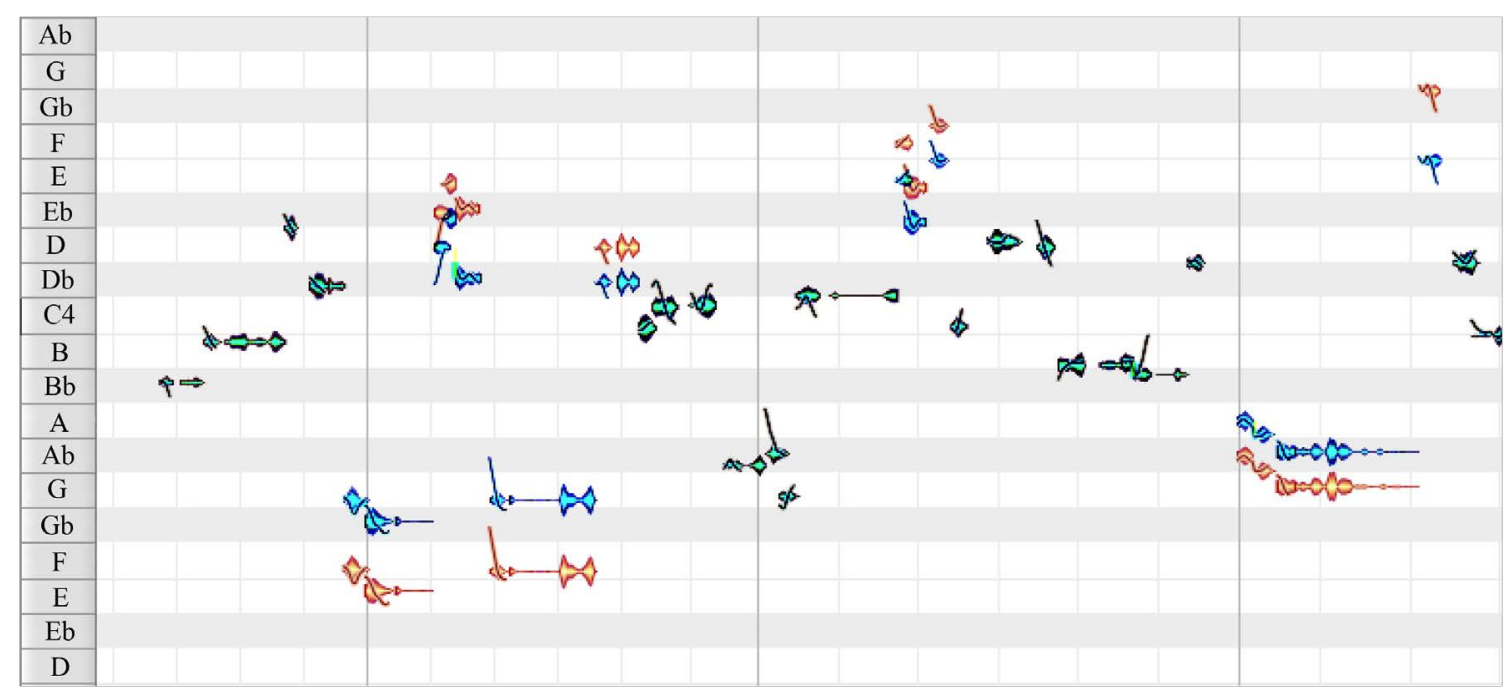

Figure 2. Melodyne screenshots of original and pitch range manipulated voices. 
Table 1. Data sets used in the study.

\begin{tabular}{ccccc}
\hline Data Sets & Gender/Sexual Orientation & $\begin{array}{c}\text { Number of } \\
\text { Recordings }\end{array}$ & $\begin{array}{c}\text { Fo Fundamental } \\
\text { Frequency Manipulation }\end{array}$ & $\begin{array}{c}\text { Pitch Range } \\
\text { Manipulation }\end{array}$ \\
\hline HEMOR & Male heterosexual original & 5 & $\times$ & $\times$ \\
HEFOR & Female heterosexual original & 5 & $\times$ & $\times$ \\
HOMOR & Male homosexual original & 5 & $\checkmark$ & $\times$ \\
HEMP & Male heterosexual pitch & 5 & $\checkmark$ & $\times$ \\
HEFP & Female heterosexual pitch & 5 & $\checkmark$ & $\times$ \\
HOMP & Male homosexual pitch & 5 & $\checkmark$ & $\checkmark$ \\
HEMPR & Male heterosexual pitch range & 5 & $\checkmark$ & $\checkmark$ \\
HEFPR & Female heterosexual pitch range & 5 & $\checkmark$ & $\checkmark$ \\
HOMPR & Male homosexual pitch range & 5 & $\checkmark$ & $\checkmark$ \\
\hline
\end{tabular}

random sampling method. These experimental sets were presented to $30(n=30 ; 15$ males, 15 females) raters in an isolated and non-echoing laboratory environment. These raters, via a survey form, were requested to rate these voice recordings using a five-point Likert scale with regard to four criteria: 1) male-female, 2) masculine-effeminate, 3) heterosexual-homosexual, and 4) real-manipulated.

In addition, an open ended question inquiring what the reasons/clues in the voice recordings were that made them to identify a recording as gay/male homosexual was asked to the raters.

\section{Findings and Discussion}

The findings obtained from the Likert scale were analysed using Fleiss’ Kappa Statistics (Fleiss, 1971; Randolph, 2005) which has been used for the comparison of evaluations belonging to two or more raters. The calculations were conducted using the Online Kappa Calculator tool (http://justusrandolph.net/kappa/). The Fleiss' Kappa scores for each criterion are presented in tables below.

Table 2 shows that the Fleiss' Kappa scores for the male-female criterion in the experiment indicates that the listeners obtained at least a moderate agreement for original voice recordings. In other words, most of the listeners agreed on the sex of the voice recordings. When the voice recordings were manipulated, the agreement level decreased significantly, from almost perfect agreement to slight agreement (heterosexual female original to heterosexual female pitch range manipulated).

Table 3 shows the Fleiss' Kappa scores for masculine-effeminate criterion in the experiment and these scores indicate a low level of agreement for almost all of experimental sets. This might be due that the listeners were not experts in linguistics or sociology and might not have known the meaning of masculinity and effeminacy.

Table 4 presents the Fleiss' Kappa scores for heterosexual-homosexual criterion, the scores also indicate a low level agreement especially in phonetically manipulated voice recordings. Fair and Moderate agreement levels for original voice recordings decreased to slight agreement in the manipulated recordings. This indicates that the differences in pitch and pitch range might signal a difference in gender group.

The Fleiss' Kappa scores for genuine-fake criterion in the experiment are given in Table 5, and show a slight decline as the voice recordings were manipulated.

When the overall Fleiss' Kappa results are examined it is seen that there is a fair agreement between the raters with regard to the gender of the speaker of the sound on the recordings. Put it differently, the raters identified the gender of the speaker on the voice recordings with a fair amount of agreement. For masculine-effeminate criterion there is slight agreement between the raters, i.e. less raters agreed on the masculinity or effeminacy of the speaker on the recording. When heterosexual-homosexual criterion is addressed, it is seen that the raters have a slight agreement on identifying the sexual orientation of the speaker. The raters also mostly agreed fairly on the genuine or fake (altered) voice recordings.

However, Fleiss’ Kappa scores, i.e. the level of agreement is not sufficient; in addition to how much the listeners agreed, we have to consider on what the listeners agreed. When we evaluate the results for each data set, it is seen in Figure 3 that the raters reached a higher rate in identifying the sex of the speaker in HEMO, HEFO, 
Table 2. Fleiss’ Kappa scores for the Male-Female criterion in the experiment.

\begin{tabular}{ccccc}
\hline Criterion & Experimental Set & Number of raters (N) & Fleiss' Kappa (к) & Interpretation \\
\hline HEMO & 30 & 0.58621 & Moderate Agreement \\
HEFO & 30 & 1.0000 & Almost Perfect Agreement \\
Male-Female & 30 & 0.53736 & Moderate Agreement \\
& HOMO & 30 & 0.146554 & Slight Agreement \\
& HEMP & 30 & 0.083335 & Slight Agreement \\
& HOMP & 30 & 0.474141 & Moderate Agreement \\
& HEMPR & 30 & 0.18391 & Slight Agreement \\
& HEFPR & 30 & 0.0890813 & Slight Agreement \\
& HOMPR & 30 & 0.359198 & Fair Agreement \\
\hline
\end{tabular}

Table 3. Fleiss’ Kappa scores for the Masculine-Effeminate criterion in the experiment.

\begin{tabular}{ccccc}
\hline Criterion & Experimental Set & Number of raters (N) & Fleiss' Kappa (к) & Interpretation \\
\hline HEMO & 30 & 0.129313 & Slight Agreement \\
& HEFO & 30 & 0.359198 & Fair Agreement \\
Masculine-Effeminate & HOMO & 30 & -0.0201138 & Poor Agreement \\
& HEMP & 30 & 0.083335 & Slight Agreement \\
& HEFP & 30 & 0.0890813 & Slight Agreement \\
& HOMP & 30 & 0.083335 & Slight Agreement \\
& HEMPR & 30 & 0.091955 & Slight Agreement \\
& HEFPR & 30 & 0.160921 & Slight Agreement \\
& HOMPR & 30 & 0.0201163 & Slight Agreement \\
\hline
\end{tabular}

Table 4. Fleiss’ Kappa scores for the Heterosexual-Homosexual criterion in the experiment.

\begin{tabular}{ccccc}
\hline Criterion & Experimental Set & Number of raters (N) & Fleiss' Kappa (к) & Interpretation \\
\hline HEMO & 30 & 0.218393 & Fair Agreement \\
& HEFO & 30 & 0.474141 & Moderate Agreement \\
HOMO & 30 & 0.0172425 & Slight Agreement \\
& HEMP & 30 & 0.109198 & Slight Agreement \\
& HEFP & 30 & 0.0603463 & Slight Agreement \\
& HOMP & 30 & 0.00574875 & Slight Agreement \\
& HEMPR & 30 & 0.11207 & Slight Agreement \\
& HEFPR & 30 & 0.100576 & Slight Agreement \\
& HOMPR & 30 & 0.00574875 & Slight Agreement \\
\hline
\end{tabular}

Table 5. Fleiss' kappa scores for the genuine-fake criterion in the experiment.

\begin{tabular}{ccccc}
\hline Criterion & Experimental Set & Number of raters (N) & Fleiss' Kappa (к) & Interpretation \\
\hline HEMO & 30 & 0.387934 & Fair Agreement \\
HEFO & 30 & 0.758625 & Substantial Agreement \\
GOMuine-Fake & 30 & 0.275864 & Fair Agreement \\
& HEMP & 30 & -0.028735 & Poor Agreement \\
& HEFP & 30 & 0.278738 & Fair Agreement \\
HOMP & 30 & 0.175289 & Slight Agreement \\
HEMPR & 30 & 0.0574725 & Slight Agreement \\
HEFPR & 30 & 0.356324 & Fair Agreement \\
HOMPR & 30 & 0.03161 & Slight Agreement \\
\hline
\end{tabular}




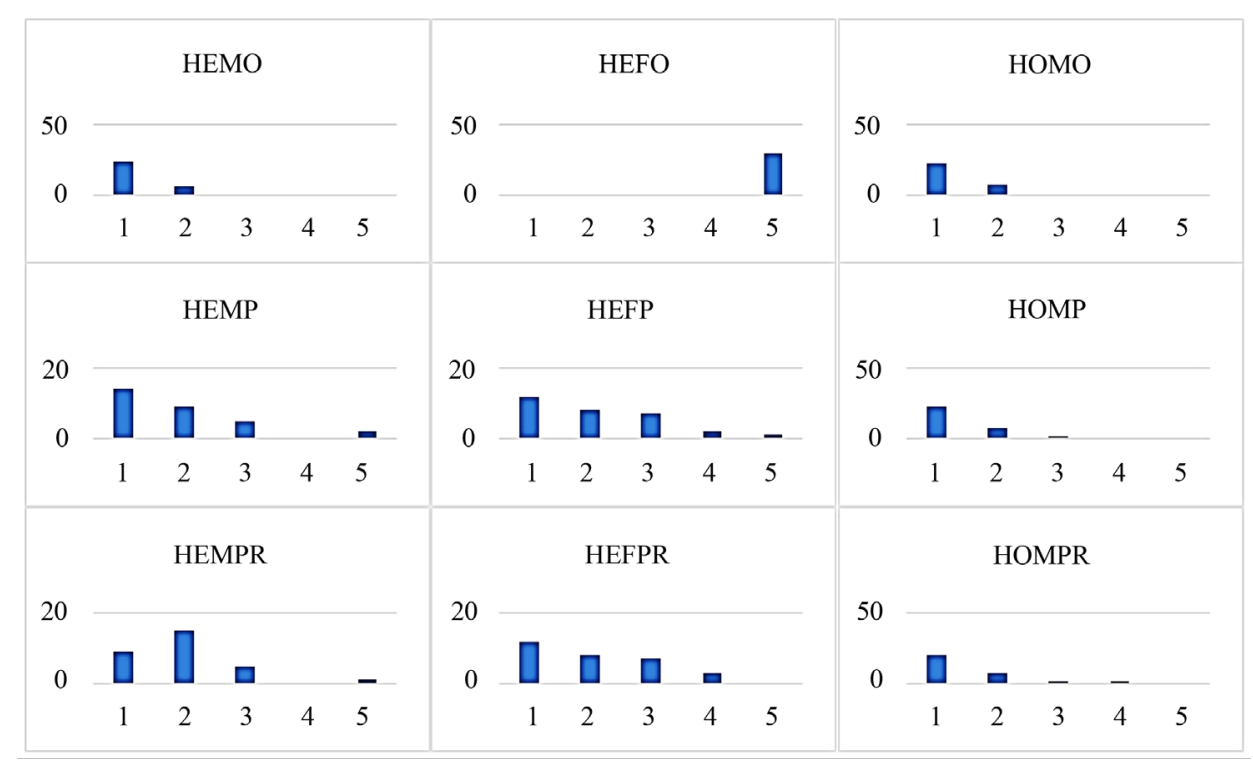

Figure 3. Listeners' evaluations of male-female criterion.

and HOMO, i.e. the original voice recordings without any manipulation. In HEMP, HEFP and HOMP in which the F0 fundamental frequency had been changed, the raters exhibited a tendency towards identifying the speakers as male. In voice recordings with both manipulated F0 fundamental frequency and manipulated pitch range (HEMPR, HEFPR, HOMPR), again the raters tended to identify the speakers as male (Figure 3).

As for the heterosexual-homosexual evaluation presented in Figure 4, the raters had a higher accuracy in identifying the sexual orientation in original voice recordings without any manipulation (HEMO, HEFO, HOMO). In voice recordings with manipulated F0 fundamental frequency (HEMP, HEFP, HOMP) the raters generally identified the female voices which had been converged towards male sounds as homosexual. The raters identified the homosexual sounds which had been converged towards male voices almost equally as homosexual $(\mathrm{N}=13)$ and heterosexual $(\mathrm{N}=17)$. It is seen that in voice recordings in which the homosexual voice had been converged towards male sound with regard to both F0 fundamental frequency and pitch range (HOMPR), the number of raters who identified the sounds as homosexual decreased dramatically (Figure 4).

When an overall qualitative evaluation is performed on the responses given to the open ended question 'What are the reasons that make you identify the speakers identity as homosexual in the voice recordings you have rated as homosexual sound?' directed at the users, the reasons that influence the decisions of the raters are found as below:

- $\quad$ The prolonging of vowels/rounded vowels/syllables (vowel/syllable duration).

- $\quad$ Rounding of sound "r" (flapping, producing $[r]$ instead of $[r]$ sound).

- $\quad$ Rises and falls in the sound, moving or vibrant speech (pitch range).

- $\quad$ Softer voice, difference in stress and intonation.

- $\quad$ Flirtatious/effeminate speech.

- $\quad$ Wider [e] sound (wider acoustic vowel space).

- $\quad$ Similarities with previously heard homosexual speech.

- $\quad$ Higher speech production rate.

- $\quad$ Salient breathing at the end of the utterances (aspiration).

- $\quad$ More treble voice (higher F0 fundamental frequency).

- $\quad$ Exaggerated stressing.

- $\quad$ Producing [n] sound and $\{-$ yor $\}$ (progressive aspect) morpheme more stressed (hyperarticulation).

\section{Conclusion}

When the results of the Likert scales and the responses given to the open ended question are evaluated in combination the main argument of our study, which is one of the most important acoustic and phonological features 


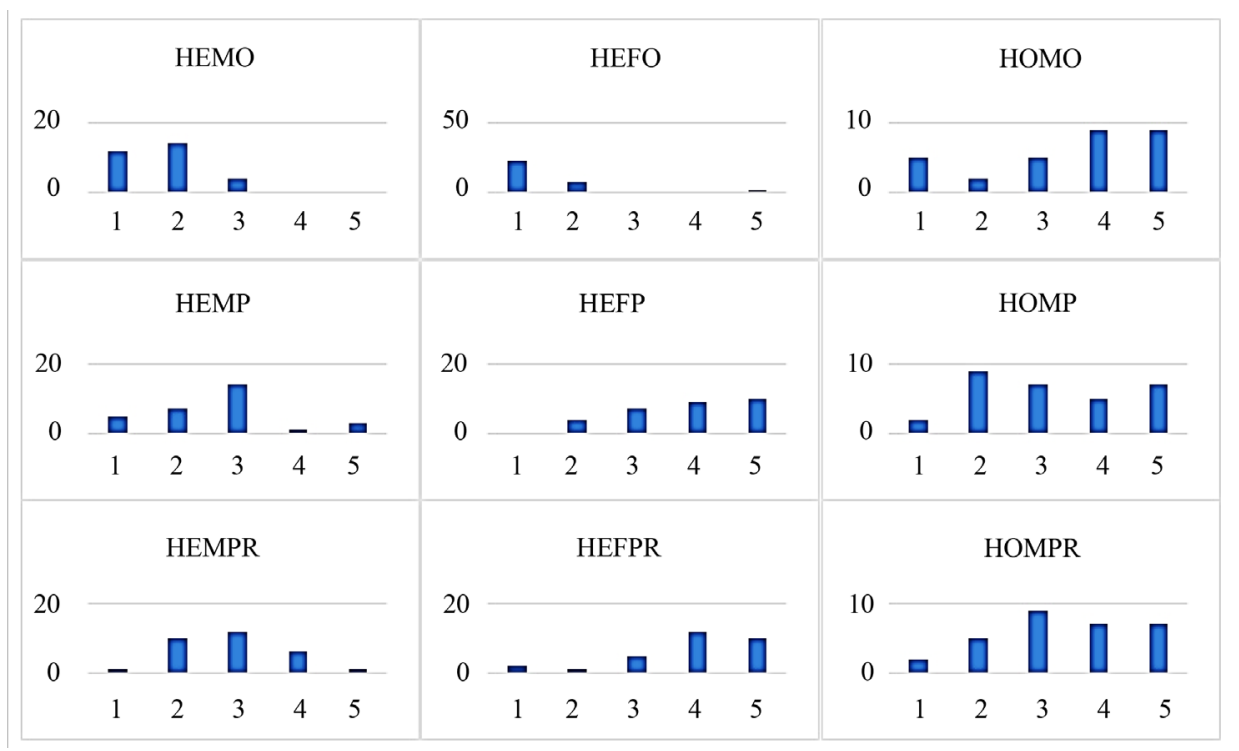

Figure 4. Listeners' evaluations of heterosexual-homosexual criterion.

that differentiate male homosexual speech from male and female heterosexual speech is pitch range/intonation, has been confirmed. We believe that we can assert that the present study would be a pioneer for the studies that would attempt to identify the features differentiating the male homosexual speech from male and female heterosexuals whose mother tongue is Turkish. Also, it can be said that the study with its methodology lays a foundation for further studies. However, the results of this study cannot, yet, be generated to all population since the sample of this research is too limited due to the sensitivity, even the taboo status of transgenderism and homosexuality in Turkish society. Future studies may include the investigation of the acoustic or phonological features identified by the raters of this study (e.g. vowel/syllable duration, flapping, hyperarticulation, wider acoustic vowel space, etc.) and also other features such as lexical and grammatical choices these individuals make during their speech. Also the further studies should be conducted with more sound recordings obtained from more participants.

\section{References}

Fleiss, J. L. (1971). Measuring Nominal Scale Agreement among Many Raters. Psychological Bulletin, 76, 378-382. http://dx.doi.org/10.1037/h0031619

Gaudio, R. P. (1994). Sounding Gay: Pitch Properties in the Speech of Gay and Straight Men. American Speech, 69, 30-57. http://dx.doi.org/10.2307/455948

GLAAD (2010). Gay and Lesbian Alliance against Defamation. GLAAD Media Reference Guide (8th ed.). Transgender Glossary of Terms “GLAAD”, USA.

Kugle, S. S. A.-H. (2010). Homosexuality in Islam: Critical Reflection on Gay, Lesbian, and Transgender Muslims. Oxford: Oneworld Oxford.

Kulick, D. (1999). Transgender and Language: A Review of the Literature and Suggestions for the Future. GLQ: A Journal of Lesbian and Gay Studies, 5, 605-622.

Levon, E. (2006). Hearing Gay: Prosody, Interpretation and the Affective Judgment of Men’s Speech. American Speech, 81, 56-78. http://dx.doi.org/10.1215/00031283-2006-003

Levon, E. (2007). Sexuality in Context: Variation and the Sociolinguistic Perception of Identity. Language in Society, 36, 533-554. http://dx.doi.org/10.1017/S0047404507070431

Linville, S. E. (1998). Acoustic Correlates of Perceived versus Actual Sexual Orientation in Men’s Speech. Folia Phoniatricaet Logopaedica, 50, 35-48. http://dx.doi.org/10.1159/000021447

Medhurst, A. (2002). Sexuality. Tracing Desires: Sexuality in Media Texts. In A. Briggs, \& P. Cobley (Eds.), The Media: An Introduction (pp. 313-325). NY \& London: Pearson Longman.

Pepiot, E. (2012). Voice, Speech and Gender: Male-Female Acoustic Differences and Crosslanguage Variation in English and French Speakers. XVèmes Rencontres Jeunes Chercheurs de l'ED 268, Paris: France. 
Randolph, J. J. (2005). Free-Marginal Multirater Kappa: An Alternative to Fleiss’ Fixed-Marginal Multirater Kappa. Joensuu University Learning and Instruction Symposium, Joensuu, 14-15 October 2005.

Smyth, R., Jacobs, G., \& Rogers, H. (2003). Male Voices and Perceived Sexual Orientation: An Experimental and Theoretical Approach. Language in Society, 32, 329-350. http://dx.doi.org/10.1017/S0047404503323024 